Pacific Journal of Mathematics

ON THE DEGREE OF THE SPLITTING FIELD OF AN
IRREDUCIBLE BINOMIAL

David ANDREw Gay and WilLIam YsLas Vél 


\title{
ON THE DEGREE OF THE SPLITTING FIELD OF AN IRREDUCIBLE BINOMIAL
}

\author{
David GaY and William Yslas VÉlez
}

\begin{abstract}
Let $x^{m}-a$ be irreducible over a field $F$. We give a new proof of Darbi's formula for the degree of the splitting field of $x^{m}-a$ and investigate some of its properties. We give a more explicit formula in case the only roots of unity in $F$ are \pm 1
\end{abstract}

A formula for the degree of the splitting field of an irreducible binomial over a field $F$ of characteristic 0 was given in 1926 in the following:

Theorem (Darbi [1]). Let $\zeta_{m}$ denote a primitive $m$ th-root of unity and let $x^{m}-a \in F[x]$ be irreducible with root $\alpha$. Define an integer $k$ as follows:

$$
k=\max \left\{l: l \mid m \quad \text { and } \quad \alpha^{m / l} \in F\left(\zeta_{m}\right)\right\} .
$$

Then the degree of the splitting field of $x^{m}-a$ is $m \phi_{F}(m) / k$, where $\dot{\phi}_{F}(m)=\left[F\left(\zeta_{m}\right): F\right]$.

In $\S 1$ of this paper we give a new proof of this theorem which, with an appropriate interpretation of the symbols above, will also be valid when char $F>0$. In $\S 2$, with the aid of a theorem of Schinzel, we obtain some properties of the number $k$, defined as in (1). Finally in $\S 3$, we will express $k$ explicitly as a function of $a$ and $m$ for a field $F$ of characteristic 0 such that the only roots of unity in $F$ are \pm 1 .

1. Proof of Darbi's theorem for arbitrary' characteristic. Let char $F=p>0$ and let $m$ be a positive integer. Set $m=m_{0} p^{f}$, with $\left(m_{0}, p\right)=1$ and set $\zeta_{m}=\zeta_{m_{0}}$. Thus $\phi_{F}(m)=\phi_{F}\left(m_{0}\right)$.

Our first step is to reduce the proof of the general theorem to a proof of the separable case, that is, to the case where char $F \nmid m$. Indeed, let char $F=p>0$ and $x^{m}-a$ be irreducible over $F$ with root $\alpha$. The splitting field of $x^{m}-a$ is $F\left(\alpha, \zeta_{m}\right)=F\left(\alpha^{p f}, \alpha^{m_{0}}, \zeta_{m_{0}}\right)$, which in turn is the compositum, over $F$, of $F\left(\alpha^{p^{f}}, \zeta_{m_{0}}\right)$, a separable extension of $F$, and $F\left(\alpha^{m_{0}}\right)$, a purely-inseparable extension. Thus, if Theorem 1 were true for the separable case, $x^{m_{0}}-a$ (with splitting field $\left.F\left(\alpha^{p f}, \zeta_{m_{0}}\right)\right)$, then we would have:

$$
\left[F\left(\alpha, \zeta_{m_{0}}\right): F\right]=p^{f}\left(m_{0} \dot{\phi}_{F}\left(m_{0}\right) / k\right)=m \dot{\phi}_{F}(m) / k .
$$


We therefore assume, for the rest of this paper, that char $F$ $\nmid m$. To complete the proof we will use the following:

Lemma (Norris and Vélez, [5]). Let $x^{m}-a$ be irreducible over $F$ with root $\alpha$. Let $n=\max \left\{l: l \mid m\right.$ and $\left.\zeta_{l} \in F(\alpha)\right\}$ and suppose $K$ is a field such that $F\left(\zeta_{n}\right) \leqq K \leqq F(\alpha)$. If $l=[F(\alpha)$ : K], then $K=$ $F\left(\alpha^{l}\right)$.

Proof. Let $f(x)$ denote the irreducible polynomial that $\alpha$ satisfies over $K$. Since $\alpha^{m}=\alpha \in F \subset K$, we have that $f(x) \mid x^{m}-a$. Thus, every root of $f(x)$ is of the form, $\zeta_{m}^{i} \alpha$, for some $i$. Hence, $f(x)=\prod_{j=1}^{l}\left(x-\zeta_{m}^{i j} \alpha\right)$. The constant term of $f(x), \prod_{j=1}^{l} \zeta_{m}^{i_{j}} \alpha=\zeta_{m}^{e} \alpha^{l}$, $e=\sum_{j=1}^{l} i_{j}$, is an element of $K \subset F(\alpha)$. Also $\alpha^{l} \in F(\alpha)$, thus $\zeta_{m}^{e} \in F(\alpha)$, and by the definition of $n, \zeta_{m}^{e} \in F\left(\zeta_{n}\right) \subset K$, thus $\alpha^{l} \in K$. Now $l=$ $[F(\alpha): K]$ and $\left[F(\alpha): F\left(\alpha^{l}\right)\right] \leqq l$, since $\alpha$ satisfies the binomial $x^{l}-\alpha^{l}$ over $F\left(\alpha^{l}\right)$. Hence we must have that $F\left(\alpha^{l}\right)=K$ and $x^{l}-\alpha^{l}$ is irreducible over $K$.

To complete the proof of Darbi's theorem, let $k^{\prime}=\left[F\left(\zeta_{m}\right) \cap\right.$ $F(\alpha): F]$. It is clear that the order of the splitting field $x^{m}-a$ is $m \phi_{F}(m) / k^{\prime}$. We must show that $k=k^{\prime}$. Now, by the definition of $n$ in the above lemma, $F\left(\zeta_{n}\right) \leqq F\left(\zeta_{m}\right) \cap F(\alpha)=K \leqq F(\alpha)$, and thus, by the lemma, we have that there is an integer $l$ such that $K=$ $F\left(\alpha^{l}\right)$. Clearly, since $x^{m}-\alpha$ is irreducible, $[K: F]=m / l=k^{\prime}$. This proves the theorem since $\alpha^{l} \in F\left(\zeta_{m}\right)$ and $l=m / k^{\prime}$.

2. Some properties of the denominator $k$ and $x^{k}-a$. For irreducible $x^{m}-a \in F[x]$, let $k$ be defined as in formula (1). Set

(2) $h=\max \left\{l: l \mid m\right.$ and $x^{l}-a$ has abelian Galois group $\}$. Then it is easy to see from the proof of Darbi's theorem that there exist positive integers $t_{1}, t_{2}$ such that

(3) $h=\phi_{F}(h) t_{1}=k t_{2}$, where $t_{2} \mid t_{1}$.

We would like to derive some properties of $h, t_{1}$, and $t_{2}$. For an integer $q$, let $w_{q}$ be the number of the $q$ th-roots of unity in $F$ and $\mathscr{P}(q)$ be the set of primes dividing $q$. Then we have:

Theorem (Schinzel). A binomial $x^{m}-a \in F[x]$ has abelian Galois group iff $a^{w_{m}}=c^{m}$, for some $c \in F$.

Proof. See [6] or [7] for a proof.

From this we obtain

Proposition 1. (A) Let $x^{m}-a$ be irreducible with abelian 
Galois group. Then $x^{m}-a$ is normal and, if $p$ is a prime and $p \mid m$, then $\zeta_{p} \in F$, that is, $p(m) \leqq p\left(w_{m}\right)$. Moreover $\phi_{F}(m) \mid m$.

(B) Let $x^{m}-a$ be irreducible and $h, t_{1}$ defined as in (2) and (3). Then $p(h) \leqq p\left(w_{h}\right)$ and $t_{1} \mid w_{h}$.

Proof. (A) Suppose $p$ prime, $p \mid m$ and $\zeta_{p} \notin F$. Then $p \nmid w_{m}$. However, by Schinzel's theorem, $a^{w_{m}}=b^{m}$ for some $b \in F$. Thus $a=c^{p}$ for some $c \in F$. Consequently $x^{m}-a$ is reducible. This contradiction implies $\zeta_{p} \in F$.

To complete the proof, since $x^{m}-a$ is irreducible and normal, $F(\alpha)$ is the splitting field of $x^{m}-a$, for any root $\alpha$ of $x^{m}-a$. Thus $\zeta_{m} \in F(\alpha)$, so $F\left(\zeta_{m}\right) \subset F(\alpha)$ and $\phi_{F}(m) \mid m$.

(B) In view of (A), all we need to show is that $t_{1} \mid w_{h}$. To do this, let $\beta$ be a root of $x^{h}-a$. Then $t_{1}=\left[F(\beta): F\left(\zeta_{h}\right)\right]$. Thus, $F\left(\beta^{t_{1}}\right)=F\left(\zeta_{h}\right)$ by the lemma. Since $x^{t_{1}}-\beta^{t_{1}}$ is irreducible over $F\left(\zeta_{h}\right)$, we have that $\beta^{l} \in F\left(\zeta_{h}\right)$ iff $t_{1} \mid l$. However, by Schinzel's theorem we have $a^{w_{h}}=c^{h}$ (for some $c \in F$ ), so that $\beta=\zeta_{h}^{i} \zeta_{h w_{h}}^{j} c^{1 / h}$, for some $i, j$. Thus $\beta^{w_{h}}=\zeta_{h}^{i w_{h} \zeta_{h}^{j} c} \in F\left(\zeta_{h}\right)$, and consequently $t_{1} \mid w_{h}$.

3. Applications. In this section let $F$ denote a field with the following two properties: (a) char $F=0$, and (b) if $\zeta_{m} \in F$, then $\zeta_{m}= \pm 1$. Clearly real fields satisfy properties (a) and (b). Furthermore, $w_{m}=1$ if $m$ is odd and $w_{m}=2$ if $m$ is even.

Proposition 2. (A) The irreducible, normal binomials in $F[x]$ with abelian Galois groups are:

(i) $x-c$

(ii) $x^{2}-c, \sqrt{c} \notin F$

(iii) $x^{4}+c^{2}, c^{2} \neq 4 d^{4}, d \in F$

(iv) $x^{2^{h}}+c^{2^{h-1}}, h \geqq 3, \sqrt{2} \notin F, c \neq 0$.

(B) Relative to the irreducible binomial $x^{m}-a \in F[x]$,

(i) $h=\max \left\{2^{q}: 2^{q} \mid m\right.$ and $\left.-a=c^{2^{q-1}}, c \in F\right\}$.

(ii) $t_{1}=\left\{\begin{array}{l}1, \text { if } h=1 . \\ 2, \text { if } h>1 .\end{array}\right.$

(iii) $k=\left\{\begin{array}{l}h, \text { if } h=1 \text { or } h=2^{q},-a=c^{2^{q-1}} \text { and } \zeta_{2^{q+1}} \sqrt{c \in F}\left(\zeta_{m}\right) \text {. } \\ h / 2, \text { otherwise. }\end{array}\right.$

In particular, $k$ is a power of 2 . If $\sqrt{2} \notin F$, then any power of 2 is possible. If $\sqrt{2} \in F$, then $k=1,2$, or 4 .

Proof. (A) If $x^{m}-a$ is irreducible, normal, and abelian, then by Proposition 2 , we have that $m=2^{q}$, for some $q \geqq 0$. Schinzel's theorem then implies $a^{2}=c^{2 q}$, for some $c \in F$. Thus, if $q \geqq 1$, $a= \pm c^{2^{q-1}}$. The rest follows by Cappelli's theorem for irreducible 
binomials ([4], p. 62).

Conversely, it is easy to check that the binomials (i)-(iv) are irreducible, normal, with abelian Galois group.

(B) Statement (i) follows from (A).

To prove (ii), note first that by Proposition $2, t_{1} \mid w_{2^{q}}$. Thus $t_{1}=1$ or 2 . If $h=1$, then clearly $t_{1}=1$. Assume that $h>1$. Recall that $t_{1}=\left[F(\beta): F\left(\zeta_{2 q}\right)\right]$, where $\beta$ is a root of $x^{2^{q}}+c^{2^{q-1}}$. If $h=2$, then since $\left[F\left(\zeta_{4}\right): F\right]=2$, we must have that $t_{1}=2$. If $q>2$, then by (A) we have that $\sqrt{2} \notin F$. Hence $\left[F\left(\zeta_{2 q}\right): F\right]=2^{q-1}$, and thus $t_{1}=2$.

Finally, to prove (iii), we note that $t_{2} \mid t_{1}$ and by (ii), $t_{1}=1$ or 2 , so $t_{2}=1$ or 2 . Furthermore, if $h=2^{q}(q \geqq 1)$ then $t_{2}=1$ iff the splitting field of $x^{2 q}+c^{2^{q-1}}$ is contained in $F\left(\zeta_{m}\right)$ iff $\zeta_{2 q+1} \sqrt{c} \in F\left(\zeta_{m}\right)$.

Thus, if the $h$ of formula (2) has been determined, then

$$
k=\left\{\begin{array}{l}
h, \text { if } h=1 \text { or } \sqrt{c} \in F\left(\zeta_{2 m}\right) \\
h / 2, \text { otherwise. }
\end{array}\right.
$$

If $m=2^{l} \cdot p_{1}^{a_{1}} \cdots p_{q}^{a_{q}}$, with $l \geqq 1$ and $p_{1}, \cdots, p_{q}$ distinct odd primes, then the condition $\sqrt{c} \in F\left(\zeta_{2 m}\right)$ is equivalent to the condition $\sqrt{c} \in F\left(\zeta_{2} l+1_{P}\right)$, where $P=p_{1} \cdots p_{q}$. For $F=Q$, the latter is equivalent to $\sqrt{c} \in Q\left(\zeta_{2^{a}}\right)$, where $a=\min \{3, l+1\}$. For an arbitrary real field however, we cannot do as well. Indeed, given any integer $q \geqq 3$, there exists an integer $m$ with $2^{q} \| m$, a real field $F$ and $c \in F$ such that $\sqrt{c \notin} F\left(\zeta_{2 m}\right)$, yet $\sqrt{c} \in F\left(\zeta_{m}\right)$. (See [2], 5.4.)

Proposition 2 generalizes a theorem of Hooley ([3], pp. 212-214).

\section{REFERENCES}

1. G. Darbi, Sulla riducibilita delle equazioni algebriche, Annali di Mat. pur e Appl., Ser. 4, 4 (1926), 185-208.

2. D. Gay, On normal radical extensions of real field, to appear, Acta Arithmetica, 35 (1978).

3. C. Hooley, On Artin's conjecture, J. für Mathematik, Band 225, (1967), 209-220.

4. I. Kaplansky, Fields and Rings, Univ. of Chicago Press, 1969.

5. M. J. Norris and W. Y. Vélez, Structure theorems for radical extensions of fields, to appear, Acta Arithmetica.

6. A. Schinzel, Abelian binomials, power residues, and exponential congruences, Acta Arithmetica, 32 (1976/1977), 245-274.

7. W. Y. Vélez, On Normal Binomials, to appear, Acta Arithmetica.

Received September 16, 1977 and in revised form March 10, 1978. Supported in part by Battelle Institute and Fonds National Suisse.

UNIVERSITY OF ARIZONA

Tucson, AZ 85721 


\section{PACIFIC JOURNAL OF MATHEMATICS}

\section{EDITORS}

RICHARD ARENS (Managing Editor)

University of California

Los Angeles, California 90024

C. W. CurTis

University of Oregon

Eugene, OR 97403

C. C. MOORE

University of California

Berkeley, CA 94720
J. DUGUNDJI

Department of Mathematics University of Southern California Los Angeles, California 90007

R. FinN AND J. Milgram Stanford University Stanford, California 94305

\section{ASSOCIATE EDITORS}

E. F. BECKENBACH

B. H. NeumanN

F. WOLF

K. YoSHIDA

\section{SUPPORTING INSTITUTIONS}

UNIVERSITY OF BRITISH COLUMBIA CALIFORNIA INSTITUTE OF TECHNOLOGY UNIVERSITY OF CALIFORNIA MONTANA STATE UNIVERSITY UNIVERSITY OF NEVADA, RENO NEW MEXICO STATE UNIVERSITY OREGON STATE UNIVERSITY UNIVERSITY OF OREGON
UNIVERSITY OF SOUTHERN CALIFORNIA

STANFORD UNIVERSITY

UNIVERSITY OF HAWAII

UNIVERSITY OF TOKYO

UNIVERSITY OF UTAH

WASHINGTON STATE UNIVERSITY

UNIVERSITY OF WASHINGTON 


\section{Pacific Journal of Mathematics \\ Vol. 78, No. $1 \quad$ March, 1978}

Simeon M. Berman, A class of isotropic distributions in $\mathbf{R}^{n}$ and their

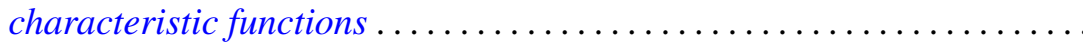

Ezra Brown and Charles John Parry, The 2-class group of biquadratic fields.

II ........................................ 11

Thomas E. Cecil and Patrick J. Ryan, Focal sets of submanifolds ....... 27

Joseph A. Cima and James Warren Roberts, Denting points in $B^{p} \ldots \ldots \ldots 41$

Thomas W. Cusick, Integer multiples of periodic continued fractions . . . . . 47

Robert D. Davis, The factors of the ramification sequence of a class of

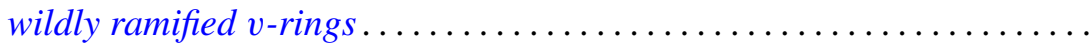

Robert Martin Ephraim, Multiplicative linear functionals of Stein

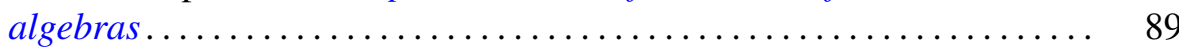

Philip Joel Feinsilver, Operator calculus . .................... 95

David Andrew Gay and William Yslas Vélez, On the degree of the splitting field of an irreducible binomial ..........................

Robert William Gilmer, Jr. and William James Heinzer, On the divisors of

monic polynomials over a commutative ring ..................

Robert E. Hartwig, Schur's theorem and the Drazin inverse .............

Hugh M. Hilden, Embeddings and branched covering spaces for three and four dimensional manifolds ............................

Carlos Moreno, The Petersson inner product and the residue of an Euler product. ...

Christopher Lloyd Morgan, On relations for representations of finite groups....

Ira J. Papick, Finite type extensions and coherence

$\mathrm{R}$. Michael Range, The Carathéodory metric and holomorphic maps on a class of weakly pseudoconvex domains ................

Donald Michael Redmond, Mean value theorems for a class of Dirichlet series

Daniel Reich, Partitioning integers using a finitely generated semigroup ...

Georg Johann Rieger, Remark on a paper of Stux concerning squarefree

numbers in non-linear sequences

Gerhard Rosenberger, Alternierende Produkte in freien Gruppen ..

Ryōtarō Satō, Contraction semigroups in Lebesgue space

Tord Sjödin, Capacities of compact sets in linear subspaces of $\mathbf{R}^{n}$

Robert Jeffrey Zimmer, Uniform subgroups and ergodic actions of exponential Lie groups......................... 